\title{
Metástasis bilateral de húmero, en paciente con cáncer renal, tratado con inmunoterapia y cirugía. Reporte de un caso
}

\section{Bilateral Humeral Metastases, in a Patient with Renal Cancer, Treated with Immunotherapy and Surgery. Case Report}

\author{
Andrés Calvo Reyes $^{1 \odot}$ Alfonso Valenzuela ${ }^{1}{ }^{\circledR}$ Roberto Montegu ${ }^{2}$ Juan Pablo Rieutord ${ }^{2}$
}

${ }^{1}$ Hospital Clínico Mutual de Seguridad CChC, Santiago, 13 Región

Address for correspondence Dr. Andrés Calvo Reyes, MD, Av. Metropolitana, Chile

2 Equipo de Hombro y Codo, Servicio de Traumatología, Clínica Red Libertador Bernardo O’Higgins, 4848, Santiago, Estación Central, 13 Salud Providencia, Chile

\section{Resumen}

\section{Palabras claves}

- cáncer renal

- metástasis ósea

- fractura bilateral húmero

\section{Abstract}

\section{Keywords}

- renal cancer

- bone metastases

- bilateral humeral fracture
El carcinoma de células renales es el 7mo cáncer en frecuencia a nivel mundial con más de 300.000 casos nuevos al año y es la 3era malignidad genitourinaria más frecuente. El sitio más común de metástasis es el pulmón mientras que el esqueleto ocupa el segundo lugar con una frecuencia que varía entre un $20 \%$ a un $35 \%$. Se ha reportado una sobrevida de 12 meses en promedio luego de la aparición de metástasis óseas.

Reporte del caso de un paciente con diagnóstico de cáncer renal de células claras y metástasis óseas (en columna, pelvis y ambos húmeros), que evoluciona con fractura de ambos brazos. El paciente es aceptado en un ensayo clínico de tratamiento con inmunoterapia y además se somete a una reducción y osteosíntesis bilateral de húmero con clavo endomedular, logrando la consolidación de ambas fracturas y, por ende, la regresión de la enfermedad.

Renal cell carcinoma is ranked $7^{\text {th }}$ in frequent cancer worldwide with more than 3000.000 new cases per year, as well as it's ranked $3^{\text {rd }}$ in frequent genitourinary malignancy.

The most common area of metastases is lung followed by skeleton in second place. The frequency of skeleton metastases varies from $20 \%$ to $35 \%$. A survival average time of 12 months is generally observed after the appearance of bone metastases.

This case report is about a patient diagnosed with clear cell renal cancer and bone metastases (in the spine, pelvis and both humerus) that evolves with fracture of both arms. The patient undergoes a clinical trial with immunotherapy and also a reduction and osteosynthesis of both arms with intramedullary nail, achieving consolidation of both fractures and regression of the disease. received

June 5,2020

accepted

August 25, 2020
DOI https://doi.org/

$10.1055 / \mathrm{s}-0040-1719021$ ISSN $0716-4548$.
Copyright (e 2020 by Thieme Revinter

Publicações Ltda, Rio de Janeiro, Brazil
License terms

(c) $(1) \$$ 


\section{Introducción}

El carcinoma de células renales es el $7 \mathrm{mo}$ cáncer en frecuencia a nivel mundial con más de 300.000 casos nuevos al año y es la 3era malignidad genitourinaria más frecuente. ${ }^{1,2}$ Representa la mayor mortalidad dentro de los cánceres urológicos con 143.000 muertes al año aproximadamente. ${ }^{1,2}$ El carcinoma de células renales es responsable del $80-90 \%$ de los tumores renales primarios, con una sobrevida promedio a 5 años del $45 \%{ }^{3}$ Dentro de ese grupo, el cáncer renal de células claras es el más frecuente. ${ }^{4}$

Al momento del diagnóstico 1/3 de los pacientes presenta una enfermedad localmente avanzada o con metástasis, mientras otro $1 / 3$ de los pacientes sufrirá alguna metástasis en el futuro. ${ }^{3,5} \mathrm{El}$ sitio más común de metástasis es el pulmón mientras que el esqueleto ocupa el segundo lugar con una frecuencia que varía entre un $20 \%$ a un $35 \%^{3,6}$ Los sitios más frecuentemente afectados por las metástasis óseas son la columna, caja torácica, pelvis y extremidades superiores. ${ }^{7}$ En general, se ha reportado una sobrevida de 12 meses luego de la aparición de metástasis óseas, ${ }^{7,8}$ aunque la llegada de tratamientos dirigidos como la inmunoterapia, pareciera mejorar ese pronóstico. ${ }^{9}$ El compromiso del sistema esquelético deriva en una serie de complicaciones secundarias al comportamiento agresivo y lítico de la metástasis. ${ }^{10}$ Dentro de esas complicaciones, destacan la hipercalcemia, compresión de médula espinal y de raíces nerviosas, como también fracturas en hueso patológico. ${ }^{11}$

$\mathrm{Al}$ analizar las fracturas en hueso patológico que afectan los huesos largos, el humero da cuenta del 16\% al 39\% de los casos. $^{12,13}$ Se describe en la literatura que las fracturas diafisiarias del húmero secundarias a una metástasis ocurren en etapas avanzadas de la enfermedad, ${ }^{14}$ generando un gran impacto en la calidad de vida de los pacientes producto del dolor, pérdida de la funcionalidad de la extremidad y dependencia para realizar las actividades cotidianas del día a día. Lo mencionado anteriormente toma especial relevancia en este caso, dado que el paciente presenta metástasis en ambos humeros, lo cual es extremadamente poco frecuente. ${ }^{15}$

A continuación, presentaremos el caso clínico de un paciente con fractura en hueso patológico de ambos húmeros, secundario a metástasis renal, sometido a un ensayo clínico con inmunoterapia en combinación con la reducción y la osteosíntesis de ambos brazos con clavo endomedular.

La publicación de ese caso, fue aprobada por el paciente. Ninguno de los autores tiene conflictos de interés que declarar.

\section{Caso Clínico}

Paciente masculino de 60 años, sin antecedentes médicos previos. En enero 2018 luego de presentar cuadro clínico caracterizado por dolor abdominal y baja de peso de un mes de evolución se diagnosticó, mediante TAC y biopsia, cáncer renal de células claras con metástasis vertebral y pélvica. Luego de 3 meses, sin haber recibido tratamiento previo, ingresó a un ensayo clínico con inmunoterapia que consistía en la administración de Pembrolizumab + Epacadostat. En

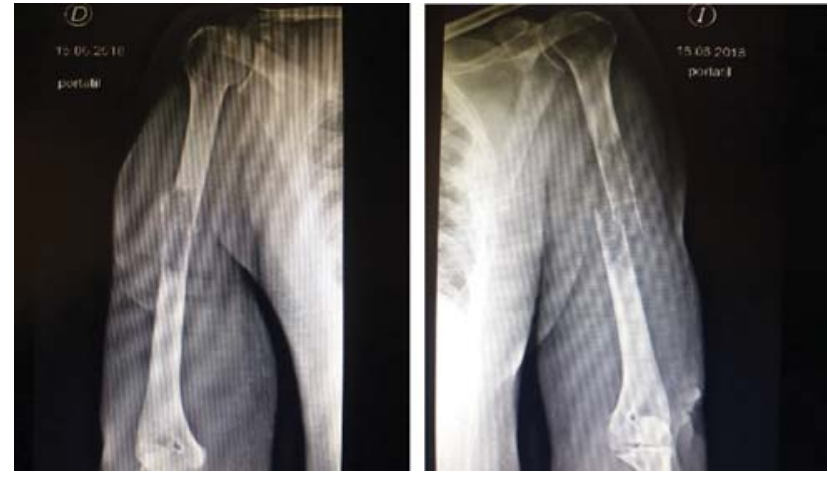

Fig. 1-2 Rx AP de húmero derecho e izquierdo, se evidencia una fractura en hueso patológico en ambos húmeros.

junio del 2018 se solicitó evaluación por traumatología porque el paciente presentaba dolor en ambos brazos luego de realizar un movimiento de rotación interna sin carga y sin antecedente de trauma. Se realizó estudio con radiografía de húmero bilateral evidenciándose fractura en hueso patológico de ambos húmeros (-Figura 1-2).

Se discutió el caso con el paciente y equipo tratante, en ese momento la expectativa de vida del paciente no superaba los 6 meses. Se decidió realizar una reducción y osteosíntesis bilateral con clavo endomedular anterógrado. En junio 2018 se sometió al paciente a cirugía, al momento de la intervención llevaba 2 meses en inmunoterapia.

Se realizó el procedimiento en un solo tiempo anestésico, paciente en posición "silla de playa". Primero se intervino el húmero derecho con una técnica anterógrada abierta para la inserción de un clavo endomedular T2 de Stryker ${ }^{\circledR}$ cuyo punto de entrada es más lateral. Se realizó una reducción cerrada y bloqueo distal con apoyo de radioscopía. Posteriormente, se preparó un nuevo campo quirúrgico para intervenir el húmero izquierdo, siguiendo exactamente el mismo procedimiento y utilizando el mismo implante. Tiempo quirúrgico total de 2 horas. El paciente quedó con cabestrillo simple bilateral.

Se realizó controles con radiología después de la cirugía, a los 6 meses y al año de evolución.

Las siguientes imágenes corresponden al control radiológico post intervención (-Figura 3-4).
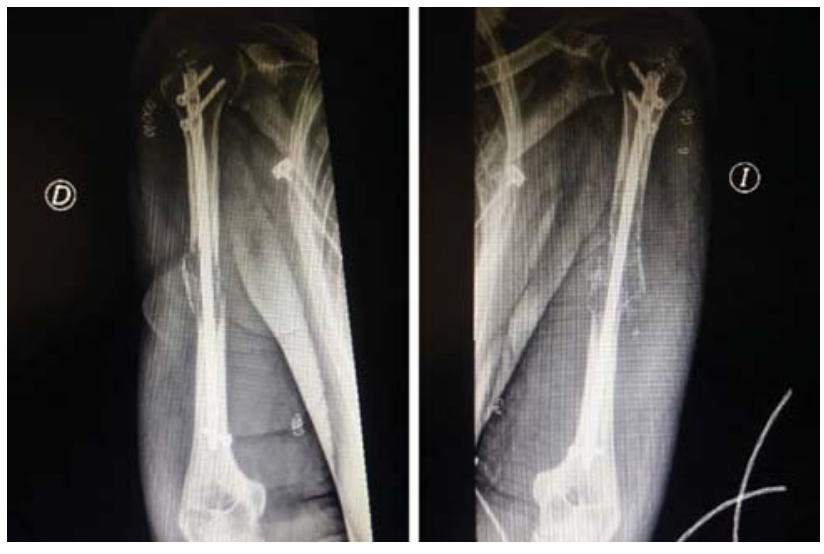

Fig. 3-4 Control post operatorio. 

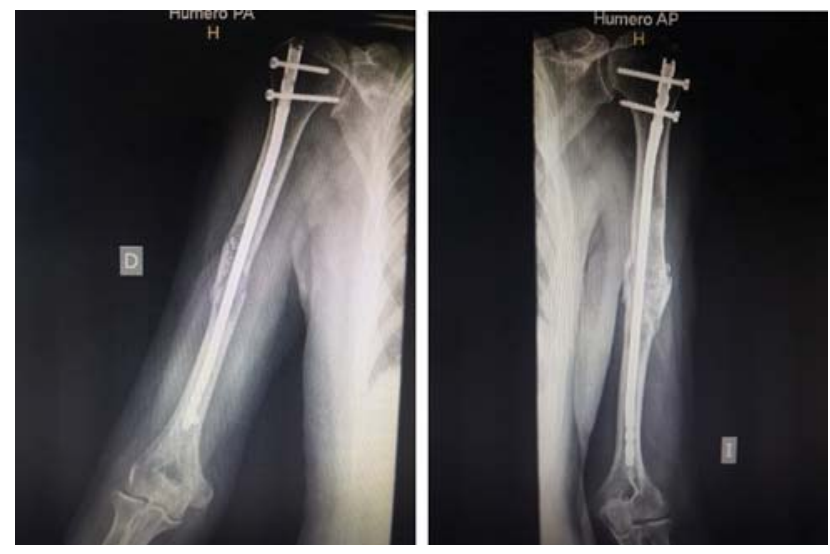

Fig. 5-6 Sexto mes de evolución.

Control radiológico a los 6 meses de evolución ( - Figura 5-6)

En este control el paciente presentaba una evolución favorable, en las imágenes se aprecian signos de consolidación y también que el 2do tornillo de bloqueo en el húmero derecho se encontraba prominente, generando molestias en el rango de movilidad del paciente junto a signos de Neer y Hawkins positivos en dicho hombro, por lo cual, se decidió reintervenir para su retiro.

Control radiológico al año de evolución (- Figura 7-8).

Las imágenes evidenciaban consolidación de la fractura en ambos húmeros.

A la fecha, ya han pasado 31 meses desde el diagnóstico de cáncer renal. Los estudios evidencian regresión de las metástasis óseas y del tumor primario. Al examen físico el paciente presenta ROM completo en ambas extremidades superiores; signos de Neer, Hawkins y Jobe negativos en ambos hombros, con un Constant score bilateral de 80 puntos y un SSV bilateral de $85 \%$.

\section{Discusión}

El tratamiento conservador de las fracturas patológicas por metástasis humeral con inmovilización de la extremidad afectada tiene malos resultados, en razón de una disminución insuficiente del dolor y baja funcionalidad. ${ }^{16,17}$ Dado lo anterior, el tratamiento quirúrgico de las fracturas
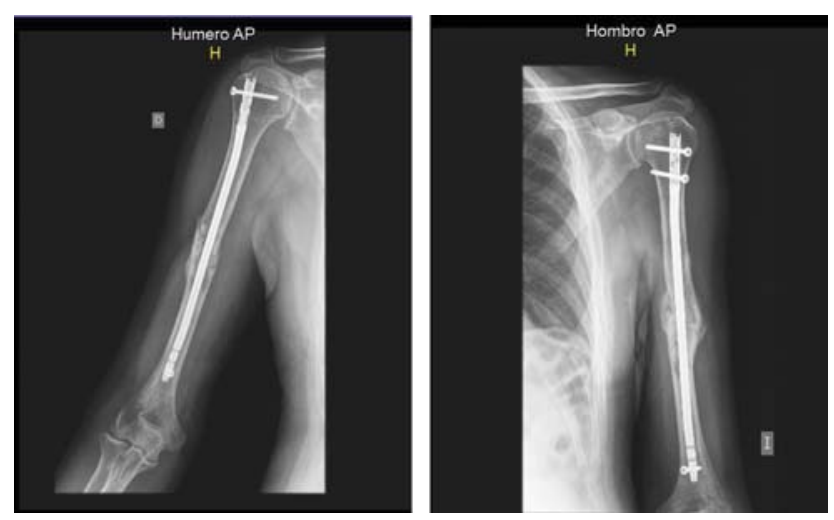

Fig. 7-8 Doce meses de evolución. diafisiarias de húmero, secundarias a metástasis, se ha convertido en la alternativa de elección. ${ }^{18}$ El objetivo del tratamiento quirúrgico es lograr, lo antes posible, disminuir el dolor y recuperar la movilidad del brazo, preferentemente sin tener que reintervenir al paciente.

Para el manejo de las fracturas de diáfisis humeral, como es el caso reportado en este trabajo, se han descrito diferentes técnicas como reducción abierta y fijación interna con placas combinadas con cemento, clavos bloqueados endomedulares o resección y reconstrucción protésica intercalar, entre otras. ${ }^{13,16,19,20}$ Cada técnica presenta ventajas y desventajas, por lo cual la decisión de qué elemento de osteosíntesis usar, debe ser analizada en un grupo multidisciplinario de especialistas, teniendo en cuenta el pronóstico del paciente, el número de lesiones, la necesidad de una cirugía menos invasiva por las condiciones generales del paciente y por supuesto, la opinión y expectativas del paciente en cuestión.

Una alternativa mencionada anteriormente es realizar la resección de la metástasis asociada a una reducción abierta y osteosíntesis con placa. Ese método, permite el control del tumor y la consiguiente estabilidad. ${ }^{21}$ Sin embargo, requiere un abordaje extenso con potencial lesión neurológica, pérdida de sangre, infección y retraso en la cicatrización de la herida. ${ }^{13}$

Por otro lado, el tratamiento con clavo endomedular es una alternativa ampliamente utilizada. ${ }^{12,16,17}$ Permite controlar las fuerzas de angulación, rotación, distracción y compresión, ${ }^{14}$ presenta menores complicaciones locales y menor tiempo quirúrgico. ${ }^{9,13,14,22}$ Sin embargo, deja la masa tumoral in situ, por lo cual es recomendado realizar radioterapia adyuvante postcirugía. ${ }^{14,16}$

Teniendo en cuenta todos los factores anteriormente mencionados, en general, la mayoría de los estudios coinciden en tratar las fracturas por metástasis en la diáfisis humeral mediante el uso de un clavo endomedular, ${ }^{9,13,14,19,22,23}$ incluso en estudios donde se obtuvo una mayor tasa de fallo de la osteosíntesis con el clavo intramedular. ${ }^{13}$

Al revisar la literatura en búsqueda de información respecto a fracturas bilaterales de humero secundarias a metástasis, la evidencia publicada hasta el mejor de nuestros conocimientos, es escasa. Wedin y col., ${ }^{23}$ analizó 208 pacientes con fracturas humerales producto de metástasis y encontró solo 6 casos de compromiso bilateral. Sin embargo, no describen cúal era el cáncer primario en esos 6 casos y tampoco si las fracturas de ambos humeros fueron sincrónicas o no. Casadei y col., ${ }^{9}$ estudió a 56 pacientes con diagnóstico de cáncer real y metástasis humeral, pero no describen la presencia de metástasis bilaterales. Otros estudios publicados son el reporte de casos de pacientes con fractura bilateral de húmero secundaria a mieloma múltiple. ${ }^{15,24}$

En nuestro caso, la decisión de qué tratamiento realizar, fue tomada considerando que el paciente al momento de la cirugía, tenía una baja expectativa de sobrevida y también existía una necesidad imperativa de recuperar la funcionalidad de ambos brazos lo más rápidamente 
posible porque, al tener ambos húmeros fracturados, su calidad de vida estaba severamente afectada. Se buscaba privilegiar una técnica menos invasiva que permitiera un rápido alivio de síntomas, con una baja tasa de complicaciones, evitando así nuevas intervenciones. La mejor alternativa para ofrecerle a ese paciente en particular, era realizar una osteosíntesis con un clavo endomedular anterógrado. Esa técnica ofrecía una solución paliativa que permitiría al paciente mantener una mejor calidad de vida en sus últimos meses. Sin embargo, la evolución favorable del paciente con incluso consolidación de ambas fracturas, hizo que fuera necesario una segunda intervención, que no estaba programada en la decisión inicial, para retirar uno de los tornillos de bloqueo que al estar prominente generaba molestias al paciente.

Nuestra conclusión al revisar ese caso clínico es que, con la llegada de nuevas terapias para el cáncer, la expectativa de vida de nuestros pacientes puede ser mayor a lo históricamente descrito, por lo tanto, los tratamientos actuales ya no pueden ser considerados solamente como una solución paliativa. Los equipos médicos deben pensar en la posibilidad de una solución definitiva, la cual, en el caso de fracturas en hueso patológico, debe ser capaz de mantener un constructo estable por el mayor tiempo posible.

\section{Conflicto de Intereses}

Los autores declaran no tener ningún conflicto de intereses.

\section{Referencias}

1 Ferlay J, Soerjomataram I, Dikshit R, et al. Cancer incidence and mortality worldwide: sources, methods and major patterns in GLOBOCAN 2012. Int J Cancer 2015;136(05):E359-E386

2 Siegel RL, Miller KD, Jemal A. Cancer statistics, 2016. CA Cancer J Clin 2016;66(01):7-30

3 Woodward E, Jagdev S, McParland L, et al. Skeletal complications and survival in renal cancer patients with bone metastases. Bone 2011;48(01):160-166

4 Patard JJ, Leray E, Rioux-Leclercq N, et al. Prognostic value of histologic subtypes in renal cell carcinoma: a multicenter experience. J Clin Oncol 2005;23(12):2763-2771

5 Szendroi A, Dinya E, Kardos M, et al. Prognostic factors and survival of renal clear cell carcinoma patients with bone metastases. Pathol Oncol Res 2010;16(01):29-38

6 Lipton A, Colombo-Berra A, Bukowski RM, Rosen L, Zheng M, Urbanowitz G. Skeletal complications in patients with bone metastases from renal cell carcinoma and therapeutic benefits of zoledronic acid. Clin Cancer Res 2004;10(18 Pt 2):6397S-6403S

7 Toyoda Y, Shinohara N, Harabayashi T, et al. Survival and prognostic classification of patients with metastatic renal cell carcinoma of bone. Eur Urol 2007;52(01):163-168
8 Flanigan RC, Campbell SC, Clark JI, Picken MM. Metastatic renal cell carcinoma. Curr Treat Options Oncol 2003;4(05):385-390

9 Casadei R, Drago G, Di Pressa F, Donati D. Humeral metastasis of renal cancer: Surgical options and review of literature. Orthop Traumatol Surg Res 2018;104(04):533-538

10 Guidelines on renal cell carcinoma. European Association of Urology, 2017. cited 2017 Dec 30. Available from: http://www. uroweb.org/guideline/renal-cell-carcinoma

11 Zekri J, Ahmed N, Coleman RE, Hancock BW. The skeletal metastatic complications of renal cell carcinoma. Int J Oncol 2001;19(02):379-382

12 Pretell J, Rodriguez J, Blanco D, Zafra A, Resines C. Treatment of pathological humeral shaft fractures with intramedullary nailing. A retrospective study. Int Orthop 2010;34(04):559-563

13 Sarahrudi K, Wolf H, Funovics P, Pajenda G, Hausmann JT, Vécsei V. Surgical treatment of pathological fractures of the shaft of the humerus. J Trauma 2009;66(03):789-794

14 Moura DL, Alves F, Fonseca R, Freitas J, Casanova J. Treatment of Pathological Humerus-Shaft Tumoral Fractures with Rigid Static Interlocking Intramedullary Nail-22 Years of Experience. Rev Bras Ortop (Sao Paulo) 2019;54(02):149-155

15 Sharma B, Kapoor K, Verma R. Presentation and management of bilateral fracture shaft humerus in a multiple myeloma patient: An extremely rare case report and review of literature. Indian Journal of Case Reports 2018;4(03):214-217

16 Dijkstra S, Stapert J, Boxma H, Wiggers T. Treatment of pathological fractures of the humeral shaft due to bone metastases: a comparison of intramedullary locking nail and plate osteosynthesis with adjunctive bone cement. Eur J Surg Oncol 1996;22(06):621-626

17 Piccioli A, Maccauro G, Rossi B, Scaramuzzo L, Frenos F, Capanna R. Surgical treatment of pathologic fractures of humerus. Injury 2010;41(11):1112-1116

18 Capanna R, Campanacci DA. The treatment of metastases in the appendicular skeleton. J Bone Joint Surg $\mathrm{Br} 2001 ; 83(04)$ : 471-481

19 Bayram S, Özmen E, Birişik F, Kıral D, Salduz A, Erşen A. Prognostic factors affecting survival of patients with pathologic humerus shaft fractures treated with intramedullary nailing without tumor removal. J Orthop Sci 2019;24(06):1068-1073

20 Szendrői M, Antal I, Szendrői A, Lazáry Á, Varga PP. Diagnostic algorithm, prognostic factors and surgical treatment of metastatic cancer diseases of the long bones and spine. EFORT Open Rev 2017;2(09):372-381

21 Bickels J, Kollender Y, Wittig JC, Meller I, Malawer MM. Function after resection of humeral metastases: analysis of 59 consecutive patients. Clin Orthop Relat Res 2005;(437):201-208

22 Thai DM, Kitagawa Y, Choong PF. Outcome of surgical management of bony metastases to the humerus and shoulder girdle: a retrospective analysis of 93 patients. Int Semin Surg Oncol 2006;3:5

23 Wedin R, Hansen BH, Laitinen M, et al. Complications and survival after surgical treatment of 214 metastatic lesions of the humerus. J Shoulder Elbow Surg 2012;21(08):1049-1055

24 Alexa O, Veliceasa B. Multiple myeloma with bilateral humerus location. Case report. Rev Med Chir Soc Med Nat Iasi 2013;117 (01):160-164 